\title{
THE CHEMICAL MORPHOLOGY OF THE VITREOUS
}

\author{
JOHN E. SCOTT \\ Manchester
}

\begin{abstract}
SUMMARY
The water of the vitreous is stabilised by an ordered meshwork of very fine collagen fibrils that are tied together in loosely parallel bundles or sprays by bridges of sulphated glycosaminoglycan. Some fibril bundles run at right angles to other bundles. In these respects vitreous resembles a very dilute corneal stroma. The glycosaminoglycans of the vitreous (hyaluronan and chondroitin sulphate) aggregate with themselves and with each other in solution. The protein cores of the proteoglycans are attached to collagen fibrils. Thus, a three-component cross-linked structure is formed: collagen fibril $\rightarrow$ proteoglycan protein core $\rightarrow$ glycosaminoglycan chain $\rightarrow$ glycosaminoglycan chain $\rightarrow$ protein core $\rightarrow$ collagen fibril. Hyaluronan can aggregate directly with chondroitin-6-sulphate, or with aggregated glycan cross-links, thus entering into an infinite meshwork.
\end{abstract}

The early development of the eye took place in an aquatic environment. One can speculate that light-sensitive cells took up sheltered positions at the back of a cavity filled with the watery fluid of the environment. Transparent structures (the cornea) then evolved to wall off the receptors (the retina) from damage, in a comparatively constant milieu. The prime requirement of these ante-retinal structures, that they were transparent, was economically met by incorporating as much water as possible in them. Thus the vitreous is approximately $97 \%$ water. Transparency of the highly aqueous cornea (approximately $80 \%$ water) nevertheless depends on the precise arrangement of the collagen fibrils of the stroma. ${ }^{1}$ The watery milieu in the vitreous is also structured, using molecules - collagens, proteoglycan(s) (PGs) and hyaluronan (HA) - that are characteristic of cornea and other connective tissues. How this is done is less obvious than in the case of cornea. This paper discusses recently discovered ultrastructures that are probably involved.

The transparent media must remain transparent. Migration of cells or blood vessels into the tissues is undesirable, and it seems that the major glycosaminoglycan (GAG) of the vitreous, HA, can inhibit angiogenesis

Correspondence to: Professor J. E. Scott, Chemical Morphology, Chemistry Building, Manchester University, Oxford Road, Manchester M13 9PL, UK. and endothelial cell proliferation - although degradation products of HA may stimulate both activities (see West and $\mathrm{Kumar}^{2}$ for review and discussion).

Balazs's well-known model of the vitreous is based on a sparse random meshwork of fine $(\sim 10 \mathrm{~nm})$ collagen fibrils, with HA dissolved and trapped in the interstices. ${ }^{3}$ HA was supposed to be a random coil in solution, without chemical links to the collagen and, according to some, no physico-chemical links either. Recently it has become clear that there are elements of order in this random system. HA is not the only GAG in the vitreous. Chick vitreous contains chondroitin-6-sulphate (CS6) with little or no HA, as part of type IX collagen ${ }^{4}$ and there are similar molecules in mammalian vitreous. ${ }^{5,6}$ Rotary shadowing and electron microscopy of homogenates of human and bovine vitreous showed CS attached to collagen fibrils therein. ${ }^{5}$ It was suggested that, as in extracts of cartilage, type IX collagen with a CS chain was linked to chick type II collagen fibrils. By analogy with the situation in cornea ${ }^{7}$ the CS somehow kept the fibrils apart. ${ }^{5}$

Structures of intact tissues have not been similarly examined. We therefore looked at rabbit vitreous, using the critical electrolyte (CEC) techniques previously developed to see PG-collagen interactions in cornea, tendon, skin, etc. ${ }^{8}$ In these tissues PGs were regularly and specifically associated with collagen fibrils at binding sites separated by approximately $60 \mathrm{~nm}$ (i.e. the D period of the collagen fibrils). This periodic banding reflects the repeating structure within the fibril, which is demonstrable by electron microscopy after heavy metal staining. A kind of D-repeating bar code (labelled a, b, c, d, e) is then visible, against which the PG binding sites are located. In cornea the a, c, d and e sites are occupied, the first pair by keratan sulphate (KS) PGs and the latter pair by CS(DS) PGs. ${ }^{8}$

The pictures of rabbit vitreous stained with Cupromeronic blue for PGs, and uranyl acetate for collagen, showed an ordered system of collagen fibrils bridged by PG filaments (Fig. 1). The binding sites of the sulphated PGs along the collagen fibril were separated by about $60 \mathrm{~nm}$, i.e. the $\mathrm{D}$ period, in this respect resembling those found on collagen fibrils in tendon, cornea, sclera, etc. The fibril banding pattern has not been matched with the PG binding sites. Banding patterns of thin fibrils are difficult 


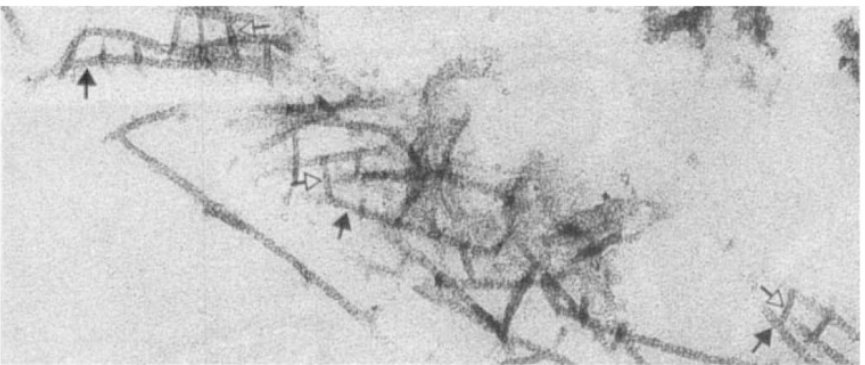

Fig. 1. Electron micrograph of rabbit vitreous, stained with Cupromeronic blue $(0.05 \% \mathrm{w} / \mathrm{v})$ in $0.025 \mathrm{~mol} / \mathrm{l}$ sodium acetate buffer pH 5.8 containing $0.3 \mathrm{~mol} / \mathrm{l} \mathrm{MgCl}_{2}$ and $2.5 \%$ glutaraldehyde. Sections were stained in uranyl acetate to demonstrate collagen fibrils. $\times 74$ 000. In $0.3 \mathrm{~mol} / \mathrm{l} \mathrm{MgCl}_{2}$ Cupromeronic blue stains only sulphated PGs; HA does not show in these conditions. PG filaments (open arrows) span the gaps between collagen fibrils (filled arrows), like the rungs in a ladder, separated by about $60 \mathrm{~nm}$. The collagen fibrils frequently are roughly parallel to each other, occurring in groups or sprays (see Fig. 2).

to establish. The fibrils were less than half the diameter of corneal fibrils $(\sim 25 \mathrm{~nm})$ and very much thinner than those of sclera $(>70 \mathrm{~nm})$.

The similar size, shape and D-periodicity of the stained PGs to those of cornea leaves open the possibility that there are small PGs in the vitreous, as there are in cornea and cartilage. The structures were unlikely to be corneal contamination, since PGs were bound at only one site within the $\mathrm{D}$ period.

The interfibrillar distances were similar to those in cornea, which, if true of the whole vitreous, implies a collagen content of about $3 \%$ wet weight, more than 10 times that measured in rabbit vitreous gel. ${ }^{3}$ However, these structures were not uniformly present throughout the vitreous. The fibrils (e.g. in human vitreous) occurred in very loose, roughly parallel bundles or sprays, separated from neighbouring bundles by considerable spaces (Fig. 2), with some bundles at right angles to other bundles. The pattern in vitreous (Figs. 1 and 2) of parallel collagen fibrils tied together by PG bridges resembles the pattern in cornea, in which parallel collagen fibrils are also bridged and tied by PGs. Cornea contains thicker and more numerous collagen fibrils, exactly parallel and at constant interfibrillar separations, in lamellae that run at right angles to each other. In many respects the vitreous resembles a very dilute corneal stroma.

Corneal PGs contain GAGs defined as dermatan sulphate (DS) because of their characteristic content of iduronic acid. It was suggested that the corneal PG bridges, or ties, were formed by side-by-side aggregations of GAG chains. ${ }^{9}$ Whether the GAG chains in the vitreous are attached to small PGs or to collagen type IX, a similar potential exists. It is proposed (by analogy with cornea) that GAG chains from adjacent collagen fibrils span the intervening space, and link with each other as duplexes or higher aggregates. DS chains have been shown to aggregate together in solution, and we have recently shown that CS6, the GAG attached to type IX collagen in chick vitreous, also self-aggregates. ${ }^{9}$

Nuclear magnetic resonance, computer dynamics simu- lations and rotary shadowing-electron microscopy indicated that aggregation is probably driven by hydrophobic bonding between the GAG polymer backbones, which, with additional hydrogen bonds, balances the electrostatic repulsion between the polyanionic aggregants. ${ }^{9}$

Other GAGs also self-aggregate, HA being the outstanding example. ${ }^{10}$ The secondary structure of HA in solution is a 2-fold helix; a flat tape-like structure, with both sides of the tape completely identical. ${ }^{9}$ HA therefore aggregates from both sides of the molecule, i.e. it is an ambidexteran. ${ }^{9}$ As a result infinite meshworks of irregular three-dimensional honeycomb form from high molecular mass HA even in the most dilute solutions $(<1 \mu \mathrm{g} / \mathrm{ml}) .{ }^{10}$ These findings were confirmed. ${ }^{5}$ Similar aggregates and meshworks were seen in homogenised mammalian vitreous.

The concept of ordered aggregation replaces the more random model of Balazs. What difference does this make to the structure/ function relationship in the vitreous?

Several corollaries follow from the demonstration that HA interacts with itself, and with similar GAGs:

1. Self-interaction can block interactions with other molecules. A pertinent experiment ${ }^{11}$ suggested that disaggregation (a kind of denaturation) of HA in solution, brought about by heating and snap-cooling, greatly increased HA inhibition of complement. This effect was reversible, on slow cooling. The aggregation mechanism could control intermolecular interactions in ways that have wide implications.

2. Infinite meshworks of high molecular mass HA are formed through flickering (i.e. reversible) contacts at large numbers of points throughout the 'mutable' structure. Small HA molecules do not form infinite meshworks, but only clumps of meshworks. ${ }^{10}$ Small HA fragments added to the infinite meshworks compete at the sticky junctions, and the meshwork begins to fall apart. ${ }^{12}$ A small amount of breakdown of HA could have a disproportionate effect on the whole gel. This may be relevant to the liquefaction of HA gels in the vitreous with age.

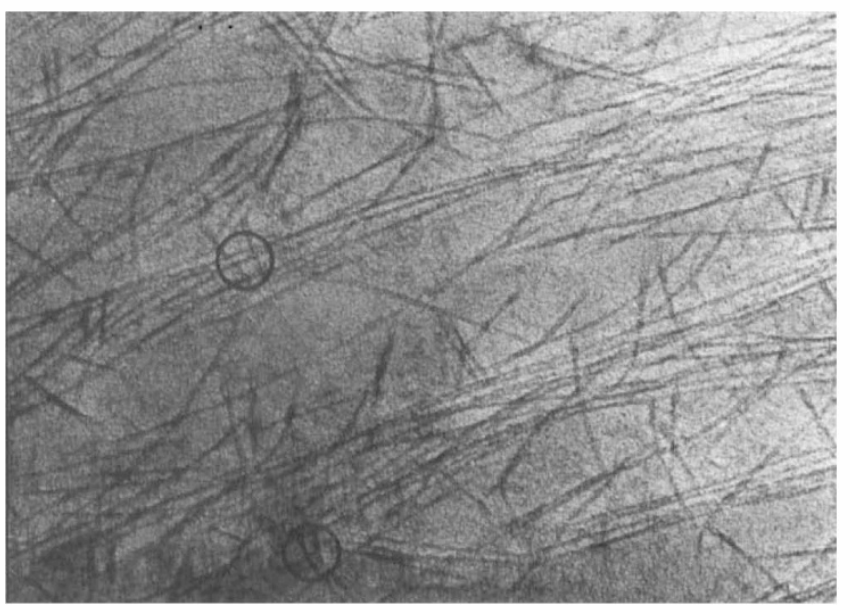

Fig. 2. Electron micrograph of human vitreous, stained with silcotungstate $\mathrm{pH} 4.5$ on the ultrathin epoxy resin section. $\times 68000$. Collagen fibrils run roughly parallel in groups of two or three or more. Some groups of fibrils are orthogonal to other groups (ringed). 


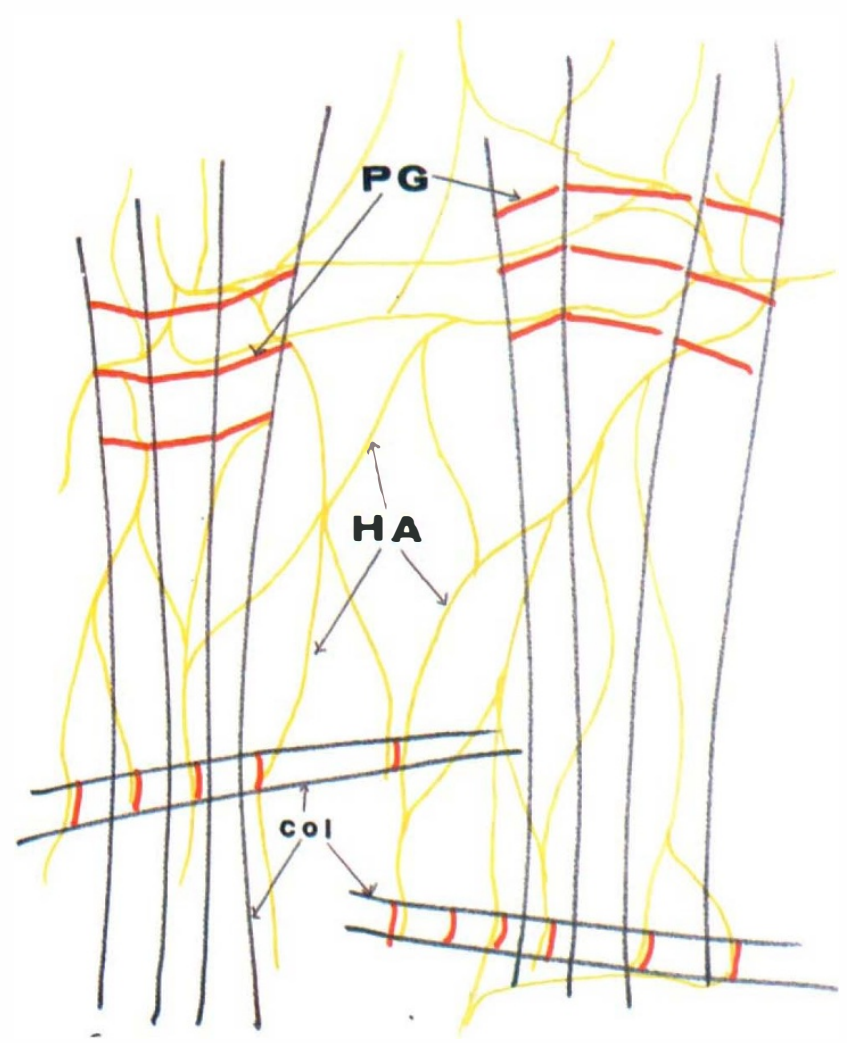

Fig. 3. Diagram of proposed three-component (collagenglycosaminoglycan-hyaluronan-glycosaminoglycan-collagen) complex which contributes, as an essentially infinite meshwork, to the semi-rigidity of mammalian vitreous humour. Black, collagen fibrils (10 nm thick); red, sulphated glycosaminoglycan (probably undersulphated CS4 and/or CS6: Scott, unpublished) component of proteoglycan (PG) as duplexes or higher aggregates; yellow, $H A$, probably in the form of highly aggregated meshworks that are able to interact with the aggregates of $C S .^{9}$

A weakness of the HA structure is its sensitivity to free radical damage, caused by the abstraction of $\mathrm{H}$ - from the C5 position of glucuronic acid, followed by depolymerisation of the HA radical. ${ }^{13}$ Damage of this kind from a small amount of radiation (perhaps even from incident light) might lead to an amplified disruption of the HA meshwork by the above mechanism.

3. HA was calculated to interact with other GAGs of similar structures - particularly CS6 and probably KS. ${ }^{9}$ CS6 has been found in mammalian vitreous (Scott, unpublished work), and the CS6 PG-collagen complex might interact with an HA meshwork via the CS6. The HA meshwork would have the role of linking every PG- collagen system with every other, thus forming an essentially endless meshwork (Fig. 3). The major connective tissue components in the vitreous would then hold together in a mutually supportive three-dimensional structure (Fig. 3), which would be semi-permanent, at least.

My thanks are due to Alison Cribb and Alan Duncan for electron microscopy, and to Dr. P. N. Bishop for the human vitreous.

Key words: Aggregation, Chondroitin sulphate, Collagen, Cornea, Cupromeronic blue, Glycosaminoglycans, Hyaluronan, Proteoglycans, Secondary structure.

\section{REFERENCES}

1. Maurice D: The structure and transparency of the cornea. $J$ Physiol 1957, 136: 263-86.

2. West DC, Kumar S: Hyaluronan and angiogenesis. Evered D, Whelan J, eds. In: The Biology of Hyaluronan. Ciba Foundation Symposium no. 143. Chichester: Wiley, 1989:187-207.

3. Balazs EA: Functional anatomy of the vitreous. Biomedical Foundations of Ophthalmology 1981, 1: 1-16.

4. Yada T, Suzuki S, Kobayashi K, Kobayashi M, Hoshino T, Horie K, Kimata K: Occurrence in chick embryo vitreous humour of a type IX collagen proteoglycan with an extraordinarily large chondroitin sulphate chain and a short a1 polypeptide. J Biol Chem 1990, 265: 6992-9.

5. Mayne R, Randolph GB, Wright DW, Ren Zhao X: Morphological and biochemical studies of the structure of the vitreous and the zonular fibres. Biochem Soc Trans 1991, 19: 868-71.

6. Bishop PN, McLeod D, Ayad S: Extraction and characterisation of the intact form of bovine vitreous type IX collagen. Biochem Biophys Res Commun 1992, in press.

7. Scott JE: Proteoglycan : collagen interactions and comeal ultrastructure. Biochem Soc Trans 1991, 19: 877-81.

8. Scott JE: Proteoglycan-fibrillar collagen interactions. Biochem J 1988, 252: 313-23.

9. Scott JE: Supramolecular organisation of extracellular matrix glycosaminoglycans, in vitro and in the tissues. FASEB J 1992, 6: 2639-45.

10. Scott JE, Cummings C, Chen Y, Brass A: Secondary and tertiary structures of hyaluronan in aqueous solution, investigated by rotary shadowing-electron microscopy and computer simulation. Hyaluronan is a very efficient meshwork-forming polymer. Biochem J 1991, 274: 699-705.

11. Chang N-S, Boackle RJ, Armand G: Hyaluronic acidcomplement interactions. I. Reversible heat-induced anticomplementary activity. Mol Immunol 1985, 22: 391-7.

12. Welsh EJ, Rees DA, Morris ER, Madden JK: Competitive inhibition evidence for specific intermolecular interactions in hyaluronate solutions. J Mol Biol 1980, 138: 375-82.

13. Scott JE: Periodate-induced viscosity decreases in aqueous solutions of acetal- and ether-linked polymers. Carbohydrate Res 1973, 28: 53-9. 